\title{
Application of a finite element method for variational inequalities
}

Mehmet Ali Akinlar

\section{"Correspondence:}

mehmetaliakinlar@gmail.com

Department of Mathematics, Bilecik

Seyh Edebali University, Bilecik,

11210, Turkey

\begin{abstract}
In this paper we explore the application of a finite element method (FEM) to the inequality and Laplacian constrained variational optimization problems. First, we illustrate the connection between the optimization problem and elliptic variational inequalities; secondly, we prove the existence of the solution via the augmented Lagrangian multipliers method. A triangular type FEM is employed in the numerical calculations. Computational results indicate that the present finite element method is a highly efficient technique in these sorts of variational problems involving inequalities.
\end{abstract}

AMS Subject Classification: $35 \mathrm{~J} 86 ; 26 \mathrm{D} 10$

Keywords: augmented Lagrangian multipliers method; variational inequalities; optimization; finite element method

\section{Introduction}

Nonlinear variational inequalities (VI) and optimal control problems are a significantly important class of problems having a broad range of applications in mathematics, engineering, mechanics and physics. Elasticity, computational fluid mechanics, computational mechanics are some of the particular applications of these types of problems.

In the present day, nonlinear variational problems or partial differential equation based optimal control problems are not only important but also very challenging problems which usually involve high storage requirements, hard optimization and solution techniques. Therefore, finding reliable and efficient computational and numerical techniques along with fast implementation methods for the solution of these types of problems is quite useful and one of active research areas in the subject. In this paper we explore the application of a finite element method (FEM) [1] to the inequality and Laplacian constrained variational optimization problems [2].

Structure of this paper can be summarized as follows. Section 2 overviews some related methods briefly. In Section 3 we express the model problem as an inequality and Laplacian constrained variational optimization problem. In the same section, we present a theorem which shows the connection between the optimization problem and the second kind variational inequality problems. We discretize the VI problems using a finite element method. We complete the paper with a conclusion section where we discuss the method presented in this paper and point some possible future extensions.

C 2013 Akinlar; licensee Springer. This is an Open Access article distributed under the terms of the Creative Commons Attribution License (http://creativecommons.org/licenses/by/2.0), which permits unrestricted use, distribution, and reproduction in any medium, provided the original work is properly cited. 


\section{Related studies}

In this section our goal is to present some related work where some type of FEM is employed as a numerical solution technique. Although there are a vast amount of related studies, we will try to mention only a few of those which have some particular and significant application areas and consider the ones which set up the connection between variational inequalities and optimal control problems besides employing FEM in numerical calculations. Before we start mentioning some of those related studies, we first illustrate an application of the present FEM to a specific variational inequality problem. Let $V=H_{0}^{1}(\Omega)$, and

$$
a(u, v)=\int_{\Omega} \nabla u \cdot \nabla v d \Omega
$$

where

$$
\nabla u \cdot \nabla v=\frac{\partial u}{\partial x_{1}} \frac{\partial v}{\partial x_{1}}+\frac{\partial u}{\partial x_{2}} \frac{\partial v}{\partial x_{2}}
$$

and $L(v)=\langle f, v\rangle$ for $f \in V^{*}=H^{-1}(\Omega)$ and $v \in V$. Let $\Psi \in H^{1}(\Omega) \cap C^{0}(\bar{\Omega})$ and $\Psi_{\left.\right|_{\Gamma}} \leq 0$. Define $K=\left\{v \in H_{0}^{1}(\Omega): v \geq \psi\right.$ a.e. on $\left.\Omega\right\}$. Then the variational inequality problem to which to present FEM is applied is defined as finding $u$ such that

$$
a(u, v-u) \geq L(v-u) \quad \text { for all } v \in K, u \in K .
$$

Detailed information about this variational inequality problem and the application of the present FEM to this problem might be obtained from [3].

In [4] Barret et al. study the existence of a solution to an open problem about a quasivariational inequality problem arising in a model for sand surface evolution. The authors first introduce a regularized mixed formulation involving both the primal and dual variables, and secondly study two methods for numerical solution of the problem. Detailed information about this study might be obtained in [4].

In [5] Burke et al. pose the Francfort-Marigo model of brittle fracture in terms of the minimization of a quite irregular energy functional. Employing a generalized AmbrosioTortorelli functional, the authors obtain a bound-constrained minimization problem, which can be expressed in terms of a variational inequality. By proposing a new FEM, the authors compute the local minimizer of the generalized functional.

In [6] Elliott et al. introduce a finite element approximation of a variational formulation of Bean's model for the physical configuration of an infinitely long cylindrical superconductor subject to a transverse magnetic field. The authors prove an error between the exact solution and the approximate solution for the current density and the magnetic field in appropriate norms. In the paper some numerical simulations for a variety of applied magnetic fields are also presented.

In [7] Bank et al. consider the application of primal-dual interior methods to the optimization of systems arising in the finite-element discretization of a class of elliptic variational inequalities. These problems lead to very large optimization problems with upper and lower bound constraints. When interior methods are applied to the discretized problem, the resulting linear systems have the same zero/nonzero structure as the finiteelement equations solved for the unconstrained case. 
As we have pointed above, although it is highly possible to extend this list of related studies, we refer the interested reader to the literature for further related work within the present paper.

\section{Main results}

Let $\Omega$ be a bounded polygonal domain in $\mathbb{R}^{2}$. We consider a model problem defined as follows.

For given $f \in L^{2}(\Omega)$, find a scalar function $\phi$ on $\Omega$ being a minimizer of the cost functional

$$
J(u):=\frac{1}{2} \int_{\Omega} \Delta u \Delta u d \Omega+\int_{\Omega}|\nabla u| d \Omega-\int_{\Omega} f u d \Omega
$$

over the convex set $K$ defined by

$$
K=\left\{u \in H_{0}^{1}(\Omega):|\nabla u| \leq 1 \text { almost everywhere on } \Omega\right\},
$$

where the Sobolev space $H_{0}^{1}(\Omega)$ is defined as follows:

$$
\begin{aligned}
& H^{1}(\Omega)=\left\{\phi \in L^{2}(\Omega), \frac{\partial \phi}{\partial x_{i}} \in L^{2}(\Omega), \forall i=1, \ldots, N\right\}, \\
& H_{0}^{1}(\Omega)=\left\{\phi \in H^{1}(\Omega), \phi=0 \text { on } \partial \Omega\right\} .
\end{aligned}
$$

Variational inequalities might be separated into two main groups as elliptic and parabolic variational inequalities. Glowinski studies these sorts of inequalities in [3] in detail. He considers the elliptic variational inequalities (EVI) as the first kind and second kind EVI and defines those in a functional context as follows.

Let $V$ denote a real Hilbert space with the inner product $(\cdot, \cdot)$ and the associated norm $\|\cdot\| . V^{*}$ is a dual space of $V, a(\cdot, \cdot): V \times V \rightarrow \mathbb{R}$ is a bilinear, continuous and $V$-elliptic form on $V \times V, L: V \rightarrow \mathbb{R}$ continuous linear functional, $K$ is a closed convex nonempty subset of $V, j(\cdot): V \rightarrow \mathbb{R} \cup\{\infty\}$ is a convex lower semi-continuous functional. The first and second kind EVI are typically defined in the following way.

The first kind EVI: find $\phi \in V$ such that $\phi$ is a solution of the problem

$$
a(\phi, v-\phi) \geq L(v-\phi), \quad \text { every } v \in K, \phi \in K .
$$

The second kind EVI: find $\phi \in V$ such that $\phi$ is a solution of the problem

$$
a(\phi, v-\phi)+j(v)-j(\phi) \geq L(v-\phi), \quad \text { every } v \in V, \phi \in V .
$$

The next lemma sets up the connection between optimization and VI problems.

Lemma 1 [3] Let $b: V \times V \rightarrow \mathbb{R}$ be a symmetric continuous bilinear $V$-elliptic form. Let $L \in V^{\prime \prime}$ and $j: V \rightarrow \mathbb{R} \cup\{\infty\}$ be a convex lower semi-continuous proper functional. Let

$$
J(v)=\frac{1}{2} b(v, v)+j(v)-L(v) .
$$


Then the minimization problem, find $\phi$ such that

$$
J(\phi) \leq J(v), \quad \text { every } v \in V, \phi \in V,
$$

has a unique solution which is characterized by

$$
b(\phi, v-\phi)+j(v)-j(\phi) \geq L(v-\phi), \quad \text { every } v \in V, \phi \in V .
$$

Proof The proof can be seen on p.7 of [3].

It is clear that we can define the following in terms of the notations of Lemma 1:

$$
\begin{aligned}
& b(u, u):=(\Delta u, \Delta u)=\int_{\Omega} \Delta u \Delta u d \Omega, \\
& j(v):=\int_{\Omega}|\nabla v| d \Omega, \\
& L(v):=\int_{\Omega} f v d \Omega .
\end{aligned}
$$

We will approximate (1) with a finite element method introduced in [3]. Assume that $\Omega$ is a polygonal domain of $\mathbb{R}^{2}$. Consider a triangulation $\mathfrak{F}_{h}$ of $\Omega$ in the following sense: $\mathfrak{F}_{h}$ is a finite set of triangles $T$ such that

$$
\begin{aligned}
& T \subset \bar{\Omega} \quad \forall T \in \mathfrak{F}_{h}, \bigcup_{T \in \mathfrak{F}_{h}} T=\bar{\Omega}, \\
& T_{1}^{\circ} \cap T_{2}^{\circ}=\emptyset \quad \forall T_{1}, T_{2} \in \mathfrak{F}_{h} \text { and } T_{1} \neq T_{2} .
\end{aligned}
$$

Here $T_{i}^{\circ}$ denotes the inner part of the corresponding triangle. Furthermore, for all $T_{1}, T_{2} \in$ $\mathfrak{F}_{h}$ and $T_{1} \neq T_{2}$, exactly one of the following conditions must hold:

(i) $T_{1} \cap T_{2}=\emptyset$,

(ii) $T_{1}$ and $T_{2}$ have only one common vertex,

(iii) $T_{1}$ and $T_{2}$ have only a whole common edge.

$h$ is the length of the largest edge of the triangles in the triangulation. Define $P_{k}$ as a space of polynomials in $x_{1}$ and $x_{2}$ of degree less than or equal to $k$, and

$$
\sum_{h}:=\left\{P \in \bar{\Omega}, P \text { is a vertex of } T \in \mathfrak{F}_{h}\right\} .
$$

The space $V=H_{0}^{1}(\Omega)$ is approximated by the family of subspaces $\left(V_{h}^{k}\right)_{h}$ with $k=1$ or $k=2$, where

$$
V_{h}^{k}:=\left\{v_{h} \in C^{0}(\bar{\Omega}),\left.v_{h}\right|_{\partial \Omega}=0 \text { and }\left.v_{h}\right|_{T} \in P_{k}, \forall T \in \mathfrak{F}_{h}\right\}, \quad k=1,2 .
$$

It is obvious that the $V_{h}^{k}$ are finite dimensional. Then the space $K$ is approximated by

$$
K_{h}^{k}=\left\{v_{h} \in V_{h}^{k}, v_{h}(P) \geq \psi(P), \forall P \in \sum_{h}^{k}\right\}, \quad k=1,2 .
$$

Notice that $K_{h}^{k}$ for $k=1,2$ are closed convex nonempty subsets of $V_{h}^{k}$. 
With these settings, the solution $\phi \in K$ is approximated by

$$
b\left(\phi_{h}, u-\phi_{h}\right)+j(u)-j\left(\phi_{h}\right) \geq L\left(u-\phi_{h}\right), \quad \text { every } u \in K_{h}, \phi \in K_{h},
$$

or equivalently,

$$
\begin{gathered}
\int_{\Omega}\left(\Delta \phi_{h} \Delta\left(u-\phi_{h}\right)\right) d \Omega+\int_{\Omega}|\nabla u| d \Omega-\int_{\Omega}\left|\nabla \phi_{h}\right| d \Omega \\
\geq \int_{\Omega} f\left(u-\phi_{h}\right) d \Omega, \quad \text { every } u \in K_{h}, \phi \in K_{h} .
\end{gathered}
$$

Using the augmented Lagrangian multipliers method, we find a discrete solution of (1) as follows. First, let us introduce the Lagrange functional

$$
\widehat{\mathcal{L}}(u, \mu)=\frac{1}{2} b(u, u)+\int_{\Omega}|\nabla u| d \Omega-\int_{\Omega} f u d \Omega+\int_{\Omega} \mu(|\nabla u|-1) d \Omega .
$$

For $r \geq 0$, an augmented Lagrangian $\mathcal{L}_{r}$ is defined by

$$
\mathcal{L}_{r}(u, \mu)=\widehat{\mathcal{L}}(u, \mu)+\frac{r}{2} \int_{\Omega}|\nabla u-1|^{2} d \Omega .
$$

Augmented Lagrangian multipliers methods for VI problems have been introduced by Glowinski and Marrocco (see [8]). Theorem 2.1 on p.168 in [3] guarantees the existence of a solution of this optimization problem.

Let us do notice that the first component $\phi_{h}$ of (2) is then the solution of the original problem (1). Using the techniques of the variational calculus, we can write that

$$
\left(\left(1+\mu_{h}\right) \Delta \phi_{h}, \Delta\left(v-\phi_{h}\right)\right)+j(v)-j\left(\phi_{h}\right)-L\left(u-\phi_{h}\right)=\left(\mu_{h}, \Delta\left(v-\phi_{h}\right)\right) \quad \forall \phi \in V_{h},
$$

or equivalently,

$$
\begin{aligned}
& \int_{\Omega}\left(\left(1+\mu_{h}\right) \Delta \phi_{h} \Delta\left(v-\phi_{h}\right)\right) d \Omega+\int_{\Omega}|\nabla u| d \Omega-\int_{\Omega}\left|\nabla \phi_{h}\right| d \Omega-\int_{\Omega} f\left(u-\phi_{h}\right) d \Omega \\
& =\int_{\Omega} \mu_{h} \Delta\left(v-\phi_{h}\right) d \Omega .
\end{aligned}
$$

We can describe the solution algorithm as follows:

(i) Choose an initial iterate $\mu_{h}$ and $\lambda \geq 0$;

(ii) Solve the linear problem $\left(\left(1+\mu_{h}\right) \Delta \phi_{h}, \Delta\left(v-\phi_{h}\right)\right)+j(v)-j\left(\phi_{h}\right)-L\left(u-\phi_{h}\right)=\left(\mu_{h}, \Delta\left(v-\phi_{h}\right)\right), \forall \phi \in V_{h}$

(iii) Update $\mu_{h}^{\alpha+1}=\max \left\{0, \mu_{h}+\alpha\left(\nabla u_{h}^{\alpha}-1\right)\right\}$ on each cell;

(iv) Set $\alpha=\alpha+1$ and go back to (ii).

\section{Conclusion}

In this note we have applied a finite element method to the inequality and Laplacian constrained variational optimization problems. Firstly, we illustrate the connection between the optimization problem and elliptic variational inequalities; and secondly, we solve the problem using the augmented Lagrangian multipliers method. In a future work, we are 
planning to apply the present method to some specific application areas including modeling with optimal control problems.

\section{Competing interests}

The author declares that they have no competing interests.

Received: 29 September 2012 Accepted: 27 January 2013 Published: 12 February 2013

\section{References}

1. Lu, Z, Chen, Y, Huang, Y: A priori error estimates for higher order variational discretization and mixed finite element methods of optimal control problems. J. Inequal. Appl. 2012, 95 (2012)

2. $\mathrm{Xu}, \mathrm{H}$ : Efficient implementation of a modified and relaxed hybrid steepest-descent method for a type of variational inequality. J. Inequal. Appl. 2012, 93 (2012)

3. Glowinski, R: Numerical Methods for Nonlinear Variational Problems. Springer, Berlin (2008)

4. Barrett, JW, Prigozhin, L: A quasi-variational inequality problem arising in the modeling of growing sandpiles. ESAIM, Math. Model. Numer. Anal. (2012, accepted). doi:10.1051/m2an/2012062

5. Burke, S, Ortner, C, Suli, E: An adaptive finite element approximation of a generalised Ambrosio-Tortorelli functional. http://www2.maths.ox.ac.uk/oxmos/reports/pdfs/oxmos29.pdf

6. Elliott, CM, Kay, D, Styles, V: Finite element approximation of a variational inequality formulation of Bean's model for superconductivity. SIAM J. Numer. Anal. 42(3), 1324-1341 (2004)

7. Bank, RE, Gill, PE, Marcia, RF: Interior Methods for a Class of Elliptic Variational Inequalities, Large-Scale PDE-Constrained Optimization. Springer, Berlin (2003)

8. Glowinski, R, Marrocco, A: Numerical solution of two-dimensional magneto-static problems by augmented Lagrangian methods. Comput. Methods Appl. Mech. Eng. 12, 33-46 (1977)

\section{Submit your manuscript to a SpringerOpen ${ }^{\circ}$ journal and benefit from:}

- Convenient online submission

- Rigorous peer review

- Immediate publication on acceptance

- Open access: articles freely available online

- High visibility within the field

- Retaining the copyright to your article 